\title{
Aplicações do Deep Learning para diagnóstico de doenças e identificação de insetos vetores
}

\section{Deep Learning applications for disease diagnosis and identification of insect vectors}

Ewerton Pacheco de Souza', Ciro Martins Gomes', Daniel Holanda Barroso', Vinícius Lima de Miranda', Rodrigo Gurgel-Gonçalves'

DOI: 10.1590/0103-11042019S211

RESUMO Deep Learning é uma técnica de aprendizado de máquina na qual o programa computacional aprende padrões diretamente a partir de imagens classificadas previamente. O presente ensaio objetivou apresentar essa técnica e algumas de suas aplicações para diagnóstico de doenças e identificação de insetos vetores para incentivar profissionais da saúde que não tenham conhecimento aprofundado em informática e que desejem utilizar a ferramenta para realizar análises automatizadas. Deep Learning tem sido aplicado para diagnóstico de câncer, fibrose cardíaca, tuberculose, detecção de parasitos como Plasmodium e Leishmania e ainda para identificação de insetos vetores. $\mathrm{Na}$ Universidade de Brasília, a técnica tem sido aplicada para desenvolver uma ferramenta para identificar lesões ulceradas de leishmaniose em diagnóstico diferencial e para detectar Leishmania em lâminas de estudos histopatológicos. Além disso, Deep Learning tem sido usado para identificar as espécies de vetores da doença de Chagas - o que é importante para auxiliar na vigilância epidemiológica. $O$ uso da tecnologia envolve desafios éticos e procedimentais que são discutidos no presente ensaio. $\mathrm{O}$ ensaio aponta perspectivas de desenvolvimento de aplicativos que auxiliem os profissionais de saúde no diagnóstico de Leishmaniose e de vetores da doença de Chagas, o que vai ao encontro dos objetivos da pesquisa translacional.

PALAVRAS-CHAVE Aprendizado profundo. Diagnóstico. Leishmaniose. Doença de Chagas.

ABSTRACT Deep Learning is a machine learning technique in which the computational algorithm learns patterns directly from images previously classified. The present essay aims to show some of its applications for clinical diagnosis and identification of insect vectors to encourage health professionals who do not have deep knowledge of computer science and who wish to use the tool to perform automated analyzes. Deep Learning has been applied to the diagnosis of cancer, cardiac fibrosis, tuberculosis, detection of parasites such as Plasmodium and Leishmania, and to identify insect vectors. At the University of Brasília, Deep Learning has been used to develop a tool to identify ulcers caused by leishmaniasis, as well as to detect Leishmania parasites. Moreover, Deep Learning was applied to identify the species of vectors of Chagas disease, an important contribution to the epidemiological surveillance of the disease. The use of Deep Learning involves some ethical and procedural issues that are discussed in this paper. Finally, the essay points out perspectives of development of apps that assist health professionals in the diagnosis of Leishmaniasis and Chagas disease vectors, which meets the goals of translational research.

1Universidade de Brasília (UnB) - Brasília (DF), Brasil.
KEYWORDS Deep Learning. Diagnosis. Leishmaniasis. Chagas Disease. 


\section{Introdução}

O diagnóstico de doenças geralmente é um processo lento e dependente de especialistas, o que pode atrasar o tratamento específico de determinadas enfermidades ${ }^{1}$. Nesse contexto, Deep Learning 2 é uma técnica promissora para análise automatizada de imagens que pode contribuir para a área médica.

A análise de imagens de interesse em saúde com Deep Learning não se limita ao uso em diagnóstico clínico. Ela pode também, por exemplo, facilitar a vigilância de insetos vetores de doenças ${ }^{3}$. Com essa técnica, é possível identificar automaticamente espécies de barbeiros - vetores da doença de Chagas -, auxiliando a prevenção e o controle dessa doença. A grande quantidade de estudos bem-sucedidos que usam Deep Learning para desenvolver ferramentas diagnósticas sugere sua viabilidade para detecção de leishmaniose cutânea, doença que apresenta ampla variedade de manifestações clínicas, o que dificulta o diagnóstico. O presente ensaio objetiva apresentar essa técnica e algumas aplicações para diagnóstico de doenças e identificação de insetos vetores para incentivar profissionais da saúde a utilizar a ferramenta para realizar análises automatizadas.

\section{Deep Learning}

Deep Learning 2 é uma técnica de aprendizado de máquina na qual o programa computacional - que constitui uma Rede Neural Convolucional (RNC) - aprende a distinguir entre imagens diferentes tal como humanos fazem: com a experiência. Por exemplo, a rede aprende o que são cães ao analisar imagens de muitos tipos deles - grandes, pequenos e de diversas raças e cores. Da mesma forma, a rede pode diferenciar gatos, onças, tigres e leões após analisar imagens previamente classificadas.

Para uma RNC aprender a diferenciar objetos, é necessário fornecer imagens classificadas $^{2}$. Na prática, arquivos contendo várias imagens de cada tipo são fornecidas ao programa. Por exemplo, para diferenciar três espécies de protozoários da malária, organiza-se um arquivo chamado Plasmodium falciparum contendo 500 imagens; um chamado P. vivax contendo 700; e outro chamado P. malariae contendo 300. Para que o aprendizado seja efetivo, é necessário fornecer fotos variadas que representem as diversas situações em que se podem encontrar os protozoários - como esfregaços sanguíneos com alta e baixa carga parasitária. $O$ aprendizado também é mais bem-sucedido quando se oferece um número maior de fotos e que apresentem boa qualidade.

O que diferencia a técnica de Deep Learning das outras técnicas tradicionais de aprendizado de máquina - como Regressão Logística e Regime de Mínimos Quadrados Comuns - é que, com ela, o programa aprende diretamente a partir de imagens. Isso ocorre de forma mais complexa para as demais técnicas ${ }^{2}$. Deep Learning 4 tem alcançado grandes avanços na resolução de problemas que desafiaram a comunidade de inteligência artificial por muitos anos. Tem-se mostrado eficaz para descobrir estruturas intrincadas em grandes bancos de imagens usando um algoritmo de backpropagation, que indica como a máquina de aprendizado deve mudar seus parâmetros internos para melhor funcionamento a cada novo ciclo de aprendizagem.

LeCun et al. ${ }^{4}$ consideram que Deep Learning será usado com muito sucesso no futuro próximo porque requer pouco 'trabalho manual de engenharia', obtendo vantagem do grande aumento atual na capacidade de processamento dos computadores. Nesse contexto, em que há padrões a serem reconhecidos, Deep Learningé uma tecnologia de uso bastante promissor - não só para imagens, mas também para sinais vitais, como eletrocardiograma, eletroencefalograma e fonocardiograma. Atualmente, existem cursos ${ }^{2}$ e livros ${ }^{5}$ que mostram como usar a técnica e os programas. Com computadores apropriados, os profissionais de saúde interessados na técnica têm ferramentas para aplicar no diagnóstico clínico ou identificação de insetos vetores de patógenos. 


\section{Aplicações de Deep Learning no diagnóstico clínico}

Deep Learning tem sido aplicado no diagnóstico de câncer ${ }^{6}$, fibrose cardíaca ${ }^{7}$, tuberculose ${ }^{8,9}$ e detecção de parasitos como Plasmodium ${ }^{\mathbf{1 0}-13}$ e Leishmania ${ }^{\mathbf{1 4}}$. A técnica tem grande potencial de benefício para a dermatologia, nas análises de imagens de lesões de pele - o que pode ser um passo importante para diagnóstico preciso $^{\mathbf{1}}$. A aplicação da técnica pode permitir diagnóstico assistido por computador, facilitando e corroborando a decisão clínica. Nesse sentido, destaca-se um estudo ${ }^{15} \mathrm{pu}-$ blicado na revista Nature no qual um grupo conseguiu treinar uma RNC para diferenciar entre tipos de câncer de pele. Nesse estudo, foram usadas 127.423 fotografias, divididas entre 757 classes de doenças. Tais imagens foram obtidas de bibliotecas públicas, provenientes de instituições de reconhecido respaldo - Isic Archive ${ }^{16}$ e Edinburgh Dermofit Library ${ }^{17}$. O estudo apresentou resultados promissores, tendo, em condições controladas e ideais, obtido nível de acerto nas classificações das lesões superior ao de dermatologistas. Os autores sugerem que dispositivos móveis equipados com aplicativos baseados em RNC podem ampliar o acesso ao diagnóstico de doenças de pele. Os resultados obtidos não sugerem a possibilidade de substituição do médico no processo de atendimento, uma vez que a tomada de decisões e de condutas não pode ser feita de forma automática; mas que o sistema pode funcionar como um registro, confirmação e guia para o diagnóstico clínico. O registro automático pode ser benéfico ainda para questões jurídicas e burocráticas.

Em outro estudo , Deep Learning foi utilizado para promover segmentação de imagens histopatológicas cardíacas, diferenciando nas lâminas as áreas que correspondem às células musculares cardíacas daquelas que correspondem a tecido conjuntivo - que, quando em excesso, caracteriza fibrose (uma condição deletéria para o músculo cardíaco). Nesse estudo, tal diferenciação foi importante para quantificar a relação entre miócitos e tecido conjuntivo, com o escopo de verificar a ocorrência de fibrose e seu percentual. Nele foram usadas 103 imagens do tipo Whole Sliding Image (WSI), que são imagens de alta resolução de lâminas histológicas, obtidas por meio de scanner. Após processamento da imagem, foi demonstrado que a RNC diferenciou na lâmina as áreas correspondentes a miócitos, tornando-se capaz de estimar o percentual de fibrose no tecido cardíaco.

Estudos em andamento na Universidade de Brasília (UnB) têm o objetivo de utilizar a tecnologia para o diagnóstico de leishmaniose, uma doença infectoparasitária negligenciada e de grande relevância, sendo considerada pela Organização das Nações Unidas (ONU) como pertence à categoria 1 de doenças emergentes não controladas ${ }^{\mathbf{1 8}}$. O estudo apresenta dois objetivos. O primeiro é diferenciar lesões ulceradas de leishmaniose de lesões ulceradas de outras doenças de pele que apresentam manifestações semelhantes. Para o treino da rede, fotos de lesões ulceradas de 300 pacientes diagnosticados com leishmaniose serão obtidas. Cada lesão será fotografada por ângulos diferentes. Quantidades similares de imagens serão obtidas para cada doença com manifestações semelhantes à leishmaniose ${ }^{19}$ (hanseníase, câncer de pele, tuberculose e micose cutânea por exemplo). Após obtenção das fotos, a rede será treinada, e sua acurácia será verificada estimando-se sensibilidade e especificidade para detecção de lesões ulceradas de leishmaniose. $\mathrm{O}$ desempenho da rede será comparado com o obtido por médicos especialistas e não especialistas e com o resultado terapêutico do tratamento.

O segundo objetivo será verificar se a RNC identificará, em fotografia de lâminas de esfregaços, as formas amastigotas de parasitos do gênero Leishmania. Um estudo mostrou resultados promissores ao treinar uma rede para identificação de promastigotas e amastigotas de Leishmania ${ }^{11}$ em lâminas de cultura. Porém, 
os autores sugerem que os resultados podem ser melhorados com bancos de dados maiores. A metodologia do trabalho na UnB, no entanto, difere da metodologia desse estudo, uma vez que o objetivo final é trabalhar com lâminas de esfregaços de pacientes que são utilizadas na detecção dos parasitos nos serviços de rotina ambulatorial. Resultados promissores permitirão o uso da RNC como ferramenta diagnóstica na prática clínica.

Há outros exemplos de esforços recentes no uso do Deep Learning como ferramenta para uso diagnóstico: Becker et al. ${ }^{\mathbf{8}}$ demonstraram a viabilidade de usar a técnica para detectar e diagnosticar tuberculose em imagens de RX de tórax; Rajaraman et al. ${ }^{10}$ e Poostchi et al.12 demonstraram como usar a ferramenta para efetuar diagnóstico de parasitos da malária a partir de imagens de esfregaço sanguíneo; Rosado et al." desenvolveram um método de identificação de patógenos da malária usando telefones celulares; Xie et al. ${ }^{\mathbf{2 0}}$ apresentaram um modelo para diferenciar nódulos pulmonares benignos e malignos em RX de tórax, demonstrando boa acurácia; finalmente, Quinn et al. ${ }^{13}$ analisaram o desempenho da RNC no diagnóstico microscópico de malária, tuberculose e ancilostomose.

\section{Aplicações de Deep Learning para identificação automática de vetores da doença de Chagas}

A doença de Chagas é uma infecção crônica e potencialmente fatal, que causa manifestações cardíacas graves. Apesar dos avanços no controle dessa doença nas Américas, em alguns países, a vigilância epidemiológica, o diagnóstico e o tratamento não são suficientes - resultando em novas infecções e manutenção de alta carga da doença ${ }^{21}$.

A melhoria dos sistemas de vigilância depende da identificação correta dos vetores, insetos da subfamília Triatominae, conhecidos como barbeiros. Foram descritas 152 espécies

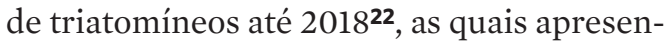
tam grande variação morfológica e diferentes capacidades vetoriais. A maioria delas mantém hábitos estritamente silvestres, enquanto outras se adaptaram a viver em ambientes antrópicos ${ }^{23}$.

A maior parte dos triatomíneos pode ser identificada utilizando caracteres morfológicos analisados por profissionais especialistas, procedimento que exige muito tempo de execução e treinamento. Portanto, há necessidade de desenvolvimento de novas estratégias de identificação desses insetos vetores com intuito de melhorar a vigilância com a participação comunitária. Nesse sentido, Deep Learning é uma ferramenta de grande potencial. Os primeiros passos para identificação automática de triatomíneos foram dados em uma pesquisa de colaboração entre a UnB, a Universidade de Kansas e o Instituto Nacional de Saúde Pública do México.

O projeto de identificação automática de triatomíneos do México e do Brasil foi baseado em uma rica base de dados obtida entre 2012 e 2017. Os resultados preliminares demonstram que triatomíneos podem ser identificados com sucesso a partir de fotografias dorsais em um processo totalmente automatizado ${ }^{3}$. Nesse trabalho, foram obtidas 2.331 imagens de 86 espécies de triatomíneos do Brasil e do México. Destas, foram incluídas nas análises somente espécies com maior número de indivíduos, totalizando 1.674 imagens de 51 espécies. O sucesso de identificação foi de aproximadamente $80 \%$ para a maioria das espécies. Em um segundo trabalho, o objetivo foi explorar os mesmos dados do primeiro artigo usando Deep Learning para promover melhorias na identificação. Para isso, foi empregado TensorFlow, uma plataforma de software de código aberto que aplica a técnica Deep Learning ${ }^{24}$. O sistema alcançou $83 \%$ e $86,7 \%$ de identificações corretas em todas as espécies mexicanas e brasileiras respectivamente - uma boa melhora em relação ao primeiro artigo de identificação automática $(80,3$ 
e $83,9 \%$ respectivamente). A incorporação de informações geográficas dos triatomíneos reduziu o número de espécies nas comparações e melhorou a proporção de identificação para $95,8 \%$ para as espécies mexicanas e para 98,9\% para as espécies brasileiras ${ }^{25}$.

O objetivo do próximo trabalho de identificação de triatomíneos é treinar uma RNC com fotografias capturadas a partir de câmeras comuns de telefones celulares - que representam a realidade do profissional que trabalhará em campo e dos cidadãos interessados em identificar um inseto parecido com um barbeiro. Espera-se que o sistema permita diferenciar triatomíneos de outros insetos similares e ainda identificar as espécies de triatomíneos ${ }^{25}$. Em uma primeira etapa experimental, as fotos que comporão o banco de imagens para treino dessa rede serão obtidas com diferentes padrões de iluminação, orientação e foco. Após elaborar o banco de imagens, a identificação dos triatomíneos será avaliada considerando os efeitos de: uso de câmeras de telefones móveis; iluminação consistente; orientação vertical exata; foco nítido; e distância do inseto para a câmera. Essas análises permitirão verificar quão robustas e confiáveis podem ser as identificações automatizadas sob circunstâncias diversas. Nossa expectativa é que o método permita uma identificação correta em diferentes condições fotográficas. Caso o resultado indique que a identificação é muito influenciada por alguma dessas variáveis, poderemos recomendar algumas orientações ou algum formato específico para realização das fotografias dos triatomíneos pela população.

\section{Desafios}

Apesar dos potenciais benefícios da tecnologia na prática clínica, algumas questões devem ser respondidas quanto ao seu uso: a população-alvo deve ser o paciente, o médico da atenção primária ou o especialista? Seria ético depender das avaliações da rede para firmar diagnósticos? Qual é o rigor que se deve adotar para o treino das redes?26.
Embora atualmente Deep Learning esteja entre as tecnologias emergentes mais relevantes $^{27}$, é importante não criar expectativas irreais sobre o tema ${ }^{\mathbf{2 8}}$. Sua incorporação na prática clínica deve ser precedida do mesmo rigor dispensado a outras intervenções médicas, com a publicação em revistas científicas e validação no contexto clínico adequado $^{26}$. É importante responder qual o papel dessa tecnologia no cuidado do paciente. Quanto a isso, situação interessante ocorreu em um estudo para avaliação de metástases linfonodais de câncer de mama ${ }^{29}$ usando Deep Learning. Nele, ficou demonstrado que a atuação conjunta dos patologistas com o uso da RCN obteve melhores resultados do que se tivesse sido utilizado só o trabalho dos especialistas ou só a $\mathrm{RCN}^{30}$. Isso demonstra o papel da técnica como ferramenta acessória no diagnóstico, não para substituir o especialista.

Espera-se que a incorporação da tecnologia apresente muitos dilemas éticos. Erros produzidos por máquinas podem ser relevantes no cuidado com a saúde ${ }^{31,32}$. Watson Oncology, um software da IBM que utiliza aprendizado de máquina, foi amplamente criticado após ser constatado que ele dava a seu usuário recomendações erradas sobre a prevenção do câncer ${ }^{31,33}$. Nesse sentido, muito se tem discutido sobre de quem é a reponsabilidade legal no caso de um algoritmo computacional levar a decisões equivocadas por parte do usuário ${ }^{34}$.

Outro dilema ético se refere às desigualdades no campo da assistência à saúde. Atualmente, a maioria dos programas de aprendizado de máquina utiliza uma base de dados que tem predominância de imagens de pacientes de pele clara. Sem imagens de pacientes melanodérmicos em quantidade suficiente para o treinamento, podem ser produzidos resultados enviesados e que não sejam aplicáveis a pacientes de origem africana ou hispânica ${ }^{35}$. Exemplificando essa situação, Wilson e colaboradores, em estudo utilizando aprendizado de máquina 
para detecção de pedestres em sistemas desenvolvidos para veículos autônomos, mostraram que os programas foram menos eficazes em detectar pedestres de pele clara que aqueles de pele escura ${ }^{36}$.

\section{Perspectivas}

A aplicação de RNC para diagnóstico clínico aumentará nos próximos anos. Quanto ao estudo desenvolvido na UnB a respeito de Leishmaniose, após comprovar a capacidade da rede em diferenciar lesões ulceradas de leishmaniose em relação a outras doenças dermatológicas, a perspectiva é testar o desempenho comparando com resultados obtidos por médicos especialistas e não especialistas. Caso os resultados sejam promissores, o estudo propõe desenvolver um aplicativo que auxilie os profissionais de saúde não especialistas no diagnóstico e encaminhamento dos pacientes, o que seria muito relevante - principalmente em regiões remotas.

Em relação à aplicação para identificação de insetos vetores, particularmente triatomíneos, a perspectiva é desenvolver aplicativo após comprovar que a RNC é capaz de classificar fotos obtidas por câmeras de telefones celulares ${ }^{25}$. Com a série fotográfica experimental a ser desenvolvida, será possível avaliar o custo em termos de perda de capacidade de identificação ao utilizar fotografias com qualidade inferior àquelas obtidas com equipamentos especializados. Com isso, espera-se que qualquer pessoa com um telefone celular em mãos possa fotografar um inseto suspeito e enviar a foto para um site a ser criado que irá processar a foto automaticamente e gerar as seguintes informações para o usuário: se o inseto é um triatomíneo e, se for, qual a sua espécie; dados relacionados com a biologia; a importância médica; e o que o morador deve fazer para acionar a vigilância.

\section{Considerações finais}

No presente estudo, foram apresentados exemplos de como Deep Learning pode auxiliar no diagnóstico de doenças - particularmente as leishmanioses -, bem como na identificação de insetos vetores da doença de Chagas, mostrando o quanto a tecnologia pode melhorar a vigilância de vetores de doenças tropicais.

No contexto de uso da ferramenta por profissionais da saúde que não tenham interesse em desenvolver diretamente uma RNC, é viável ter em um hospital uma unidade composta por técnicos capacitados na tecnologia que sejam dedicados a auxiliar os especialistas na tarefa de desenvolver as ferramentas diagnósticas de forma automatizada. Esse esforço vai ao encontro dos objetivos da pesquisa translacional: aplicar conhecimentos da pesquisa básica para as diferentes áreas clínicas (diagnóstico, tratamento e prevenção) e promover a integração entre os produtores e os usuários da pesquisa científica.

\section{Colaboradores}

Souza EP (0000-0001-9993-0520)* contribuiu com o auxílio da obtenção, edição e análise de imagens e revisão do artigo. Gomes CM (0000-0002-3069-6884)* contribuiu para a elaboração e revisão do artigo. Barroso DH (0000-0003-4128-1593)* contribuiu para a elaboração do artigo. Miranda VL (00000003-1788-0959)* contribuiu para o auxílio na obtenção, edição e análise de imagens e revisão do artigo. Gurgel-Gonçalves R (00000001-8252-8690)* contribuiu para a concepção, planejamento, análise e a interpretação dos dados; elaboração do rascunho, revisão crítica do conteúdo; e aprovação da versão final do manuscrito. 


\section{Referências}

1. Olsen TG, Jackson BH, Feeser TA, et al. Diagnostic performance of deep learning algorithms applied to three common diagnoses in dermatopathology. J. Pathol. Inform. 2018; 9:32.

2. Mathworks. Curso online: Deep learning with matlab [internet]. [acesso em 2018 out 10]. Disponível em: https://www.mathworks.com.

3. Gurgel-Gonçalves R, Komp E, Campbell LP, et al. Automated identification of insect vectors of Chagas disease in Brazil and Mexico: The Virtual Vector Lab. PeerJ. 2017; 5:e3040. Disponível em: https://peerj. com/articles/3040.pdf.

4. LeCun Y, Bengio Y, Hinton G. Deep learning. Nature. 2015; (521):436-444.

5. Zhou SK, Greenspan H, Shen D. Deep Learning for Medical Image Analysis. Elsevier; 2017.

6. Diamant I, Bar Y, Geva O, et al. Chest Radiograph Pathology Categorization via Transfer Learning. In: Zhou, SK, Greenspan H, Shen D. Deep Learning for Medical Image Analysis. Elsevier; 2017. p. 299-320.

7. Nirschl JJ, Lanowczyk A, Peyster EG, et al. Deep learning tissue segmentation in cardiac histopathology images. In: Zhou, SK, Greenspan H, Shen D. Deep Learning for Medical Image Analysis. Amsterdã: Elsevier; 2017. p. 179-195.

8. Becker AS, Bluthgen C, Phi Van VD, et al. Detection of tuberculosis patterns in digital photographs of chest X-ray images using Deep Learning: feasibility study. Int J Tuberc Lung Dis. 2017; 28(3):328-335.

9. Ghesu FC, Georgescu B, Hornegger J. Efficient Medical Image Parsing. In: Zhou, SK, Greenspan H, Shen D, organizadores. Deep Learning for Medical Image Analysis. Amsterdã: Elsevier; 2017. p. 55-81.

10. Rajaraman S, Silamut K, Hossain AM, et al. Understanding the learned behavior of customized convolutional neural networks toward malaria parasite de- tection in thin blood smear images. J Med Imaging. 2018; 5(3):034501.

11. Rosado L, Costa JMC, Elias D, et al. Automated detection of malaria parasites on thick blood smears via mobile devices. Procedia Comput Sci. 2016; (90):138144.

12. Poostchi M, Silamut K, Maude RJ, et al. Image analysis and machine learning for detecting malaria. Transl Res. 2018; (194):36-55.

13. Quinn JA, Nakasi R, Mugagga PKB, et al. Deep convolutional neural netwoks for microscopy-based point of care diagnostics. P Mach Learn Res. 2016; (56):271281.

14. Górriz M, Aparicio A, Reventós B, et al. Leishmaniasis parasite segmentation and classification using deep learning. Lect Notes Comput Sc. 2018; (10945):53-62.

15. Esteva A, Kumprel B, Novoa RA, et al. Dermatologist-level classification of skin cancer with deep neural networks. Nature. 2017; 542(7639):115-118.

16. ISIC archive - The International Skin Imaging Collaboration [internet]. [acesso em 2019 abr 1]. Disponível em: https://isic-archive.com.

17. Dermofit Image Library. High quality skin lesion images for use as a research tool in computer science and medical imaging [internet]. [acesso em $2019 \mathrm{abr}$ 1]. Disponível em: https://licensing.edinburgh innovations.ed.ac.uk/i/software/dermofit-image-library. html.

18. Vries HJC, Reedijk SH, Schallig HDFH. Cutaneous leishmaniasis: recent developments in diagnosis and management. Am J Clin Dermatol. 2015; 16(2):99-109.

19. Reithinger R, Dujardin JC, Louzir H. Cutaneous leishmaniasis. Lancet Infect Dis. 2007; 7(9):581-596.

20. Xie Y, Xia Y, Zhang J, et al. Knowledge-based collaborative deep learning for benign-malignant lung 
nodule classification on chest CT. IEEE Trans Med Imaging. 2017; 38(4):991-1004.

21. Coura JR, Viñas PA. Chagas disease: a new worldwide challenge. Nature. 2010; 465(7301): S6-S7.

22. Monteiro FA, Weirauch C, Felix M, et al. Evolution, systematics, and biogeography of the Triatominae, vectors of Chagas disease. Adv Parasitol. 2018; (99):265-344

23. Lent H, Wygodzinsky PW. Revision of the Triatominae (Hemiptera, Reduviidae), and their significance as vectors of Chagas disease. Bull Am Mus Nat Hist. $1979 ; 163: 123-520$.

24. Abadi M, Agarwal A, Barham P, et al. Tensorflow: Large-scale machine learning on heterogeneous distributed systems. Unisenix. 2016; (12):265-283.

25. Khalighifar A, Komp E, Ramsey JM, et al. Deep learning algorithms improve automated identification of Chagas disease vectors. J Med Entomol. 2019; 56:1404-1410

26. Zakhem GA, Motosko CC, Ho RS. How should artificial intelligence screen for skin cancer and deliver diagnostic predictions to patients? JAMA dermatology. 2018; 154(12):1383-4.

27. Smarter With Gartner. 5 trends emerge in the gartner hype cycle for emerging technologies, 2018 [internet]. [acesso em 2019 fev 20]. Disponível em: https:// www.gartner.com/smarterwithgartner/5-trends-emerge-in-gartner-hype-cycle-for-emerging-technologies-2018.

28. Topol EJ. High-performance medicine: the convergence of human and artificial intelligence. Nature medicine. 2019; 25(1):44-56.

29. Bejnordi BE, Veta M, Van Diest PJ, et al. Diagnostic assessment of deep learning algorithms for detection of lymph node metastases in women with breast cancer. JAMA Networks. 2017; 318(22):2199-2210.
30. Steiner DF, MacDonald R, Liu Y, et al. Impact of deep learning assistance on the histopathologic review of lymph nodes for metastatic breast cancer. Am J Surg Pathol. 2018; 42(12):1636-1646.

31. Nguyen A, Yosinski J, Clune J. Deep neural networks are easily fooled: High confidence predictions for unrecognizable images - Nguyen Deep Neural Networks. CVPR. 2015. Disponível em: https://www.cv-foundation.org/openaccess/content_cvpr_2015/papers/ Nguyen_Deep_Neural_Networks_2015_CVPR_paper.pdf.

32. Finlayson SG, Kohane IS, Chung HW, et al. Adversarial attacks against medical deep learning systems. [internet]. 2019 [acesso 2019 mar 3]. Preprint. Disponível em: https://arxiv.org/pdf/1804.05296.pdf.

33. Massdevice. Report: IBM Watson delivered 'unsafe and inaccurate' cancer recommendations [internet]. WTWH Media LLC and its licensors. [acesso em 2019 jan 6]. Disponível em: https://www.massdevice.com/report-ibm-watson-delivered-unsafe-and-inaccurate-cancer-recommendations.

34. Forbes. Can you sue an algorithm for malpractice? Interview with W. Nicholson Price [internet]. [acesso em 2019 jan 20]. Disponível em: https://www.forbes.com/sites/insights-intelai/2019/02/11/can-you-sue-an-algorithm-for-malpractice/\#524671577013.

35. Adamson AS, Smith A. Machine learning and health care disparities in dermatology. JAMA dermatology. 2018; 154(11):1247-8.

36. Wilson B, Hoffman J, Morgenstern J. Predictive Inequity in Object Detection [internet]. 2019 [acesso 2019 mar 8]. Preprint. Disponível em: https://arxiv. org/pdf/1902.11097.pdf.

Recebido em 08/04/2018

Aprovado em 21/08/2019

Conflito de interesses: inexistente

Suporte financeiro: não houve 